\title{
Docencia inversa en la asignatura Fundamentos Químicos para la Ciencia y Tecnología de los Alimentos
}

\author{
Fernando Cardona $^{\text {a,b }}$, Juan A. Llorens-Molina ${ }^{c}$ \\ ${ }^{\text {a }}$ Instituto de Biomedicina de Valencia. Consejo Superior de Investigaciones Científicas \\ ${ }^{\mathrm{b}}$ Departamento de tecnología de alimentos. E.T.S. Ingeniería Agronómica y Medio Natural. \\ Universitat Politècnica de València. \\ ${ }^{\mathrm{c}}$ E.T.S. Ingeniería Agronómica y Medio Natural. Universitat Politècnica de València. \\ juallom2@qim.upv.es
}

\section{Resumen}

Dentro del Proyecto de Docencia Inversa de la Universitat Politècnica de València (UPV), esta metodología (DI) se ha incorporado a la asignatura Fundamentos Químicos para Ciencia y Tecnología de Alimentos, para el primer curso de dicho grado. Se ha aplicado a una unidad didáctica (UD) de química orgánica, con sus prácticas de laboratorio (PL), y a las PL de la UD de química física. La herramienta fundamental ha sido Lessons, diseñada para la creación y gestión de contenidos interactivos dentro de PoliformaT (plataforma educativa on-line de la UPV). Las principales características de la UD de química orgánica han sido: la atención a los conocimientos previos, el enfoque del contenido descriptivo como trabajo autónomo y el tratamiento presencial del que posee mayor demanda cognitiva. Se ha puesto además especial énfasis en la evaluación formativa. En cuanto a las PL, se ha utilizado Lessons y Exámenes de PoliformaT para la realización de actividades prelaboratorio incluyendo un vídeo explicativo de cada práctica así como para la propuesta de actividades post-laboratorio. De este modo, la DI ha permitido optimizar las sesiones de prácticas, al prescindir de las introducciones teóricas, llegando el alumnado al laboratorio con una idea más clara de sus fundamentos teóricos y objetivos. En general, se han observado cambios positivos en el ambiente del aula, con una mayor autonomía, participación y responsabilidad del alumnado sobre su propio aprendizaje y una cierta mejora en los resultados académicos, debida probablemente a una mayor motivación.

Palabras clave: Docencia inversa, Química, Prácticas de laboratorio, PoliformaT 


\section{Introducción}

\subsection{Características generales}

La docencia inversa (DI) puede definirse como una estrategia educativa donde la clase presencial es un espacio de aprendizaje activo centrado en las tareas de mayor demanda cognitiva, en las que juega un papel esencial la interacción entre los estudiantes y entre estos y el profesorado (Tucker, 2012; Akçayir y Akçayir, 2018). Tradicionalmente, la docencia ha estado centrada en la transmisión de conocimientos en el aula y su revisión y aplicación práctica en tareas no presenciales. Por el contrario, en la DI, una determinada información conceptual y metodológica es proporcionada mediante objetos de aprendizaje diversos para que el alumno los trabaje de manera autónoma. Este aprendizaje proporciona el fundamento para las tareas presenciales, de mayor complejidad y demanda cognitiva. De este modo, el alumnado adquiere un mayor protagonismo en su formación.

En el diseño y aplicación de los materiales para el trabajo autónomo (no presencial) es importante utilizar la amplia gama de recursos tecnológicos actualmente disponibles para transmitir información: vídeos, screencasts, etc., así como diferentes herramientas interactivas: wikis, foros, etc., que hacen la clase más eficaz y amena, preparando emocionalmente al alumnado para estar más receptivos al aprendizaje. Ello es posible en el marco de plataformas de aprendizaje on-line como PoliformaT, utilizada en el Proyecto de Docencia Inversa de la Universitat Politècnica de València (UPV), (2019).

\subsection{El papel del profesorado en la docencia inversa}

Desde una perspectiva general, la DI puede situarse, de acuerdo con Akçayir y Akçayir (2018), en un enfoque del aprendizaje centrado en el estudiante y no en el profesorado. De esta manera, la organización y el contenido de las sesiones presenciales permiten una atención individualizada a los estudiantes y una mayor interacción entre ellos (Tucker, 2012), creando un espacio de aprendizaje colaborativo basado en el trabajo en pequeños grupos y en el papel del profesorado como facilitador del aprendizaje. Es posible así proporcionar un feed-back mucho más inmediato y llevar a cabo una evaluación formativa más eficaz. Abeysekera y Dawson (2015) añaden, como elemento esencial, la responsabilidad del estudiante en su propio aprendizaje, lo cual exige prestar una especial atención al desarrollo de la actividad metacognitiva del estudiante. Este factor, uno de los principales rasgos de identidad de la orientación constructivista del aprendizaje, es decisivo a la hora de implementar la DI, sobre todo en lo que concierne a las actividades no presenciales. También se refieren los citados autores al potencial que posee la preparación de los materiales de aprendizaje en cuanto al desarrollo profesional del docente, ya que le exigen un esfuerzo de 
síntesis y clarificación que repercute positivamente en la calidad de su trabajo y en su propia formación.

\subsection{La aplicación de la clase inversa: aspectos positivos y problemáticos}

La evaluación de los resultados de la DI ha sido objeto de numerosas investigaciones basadas en estudios comparativos entre grupos control y experimentales, algunas de ellas relacionadas con el aprendizaje de la química (Ryan y Reid, 2015) y particularmente con la química orgánica (Cornier y Voisard, 2018) así como con las prácticas de laboratorio (Teo et $a l ., 2014)$. En general, en todas ellas se constata un efecto positivo en el rendimiento académico y sobre todo en la motivación e implicación de los estudiantes. En cualquier caso, como puntualizan Akçayir y Akçayir (2018), no debe olvidarse que la implementación de la DI no pretende exclusivamente el logro de mejores resultados académicos, sino también facilitar la atención individualizada y la posibilidad de crear más oportunidades para aplicar los conocimientos adquiridos. Una conclusión prácticamente general de las citadas investigaciones es que el vídeo es el recurso más eficaz para presentar la información (Jensen et al., 2018), sobre todo cuando se trata de vídeos cortos para tratar conceptos concretos, tal como describen Cormier y Voisard (2018), en el contexto de la química orgánica. La eficacia de su aplicación didáctica puede explicarse partiendo de la teoría de la codificación dual (Clark y Paivio,1991), que reconoce la efectividad de la combinación de los textos verbales y la imagen. La teoría motivacional de Keller (Galicia-Alarcón y Edel-Navarro, 2014) también explica el éxito del vídeo como material de aprendizaje, ya que es un recurso capaz de integrarse en la vida cotidiana del estudiante por tratarse de un formato que le resulta muy familiar por su amplio uso en las redes sociales. Los requisitos que deben cumplir estos vídeos han sido ampliamente descritos en la literatura y el impacto de su calidad en la aplicación de la docencia inversa es también ampliamente reconocido (Akçayir y Akçayir, 2018). Estos criterios de calidad concuerdan ampliamente con las características del denominado formato Polimedia, vídeos de corta duración elaborados como objetos de aprendizaje dentro del proyecto de Docencia en Red de la UPV (“¿Qué es Polimedia?”, 2019). De hecho, existe un elevado consenso acerca del papel positivo de la aparición de una persona transmitiendo la información, tanto por la motivación que genera como por el estímulo de la atención. Otra aportación interesante del vídeo es su capacidad para facilitar el repaso previo a las pruebas de evaluación, valoración constatada en anteriores estudios (Jensen et al., 2018).

En general, puede apreciarse un cierto consenso acerca de la mejoría más acusada que la DI produce en los estudiantes con mayores dificultades iniciales (Jensen et al., 2018). Asimismo, en cuanto a las competencias transversales, se considera que la DI promueve la autonomía y 
el pensamiento crítico, tan importantes en el aprendizaje a lo largo de la vida y en el futuro desempeño profesional (O’Flaherty y Phillips, 2015).

En cuanto a las prácticas de laboratorio, la utilidad de la DI es afirmada por Teo et al. (2014), destacando la efectividad de su presentación mediante vídeos con un narrador, seguidos de cuestiones prelaboratorio, También se refieren dichos autores a una mejora en la comprensión de la fundamentación teórica y del objetivo de cada etapa experimental, así como al ahorro de tiempo que supone prescindir de la introducción teórica en el laboratorio, permitiendo la discusión final de los resultados obtenidos. Este modelo es, por otra parte, coincidente con el que los autores de este trabajo vienen aplicando habitualmente en la UPV.

Pese al balance generalmente positivo reflejado en la experimentación de la DI, diferentes retos, dificultades y dudas son también descritos en la literatura (Akçayir y Akçayir, 2018). En primer lugar, la necesidad de adaptación de los materiales didácticos, que supone un esfuerzo adicional al profesorado, sobre todo al comienzo de su implementación. Desde el punto de vista de los estudiantes, la literatura consultada coincide en señalar como el principal obstáculo el diferente grado de implicación de los estudiantes, manifestado en la falta de preparación del trabajo no presencial (Jensen et al., 2018). También hay referencias específicas a las dificultades de adaptación a la DI del alumnado del primer curso de sus estudios universitarios (O'Flaherty y Phillips, 2015).

\section{Desarrollo de la innovación}

La propuesta descrita a continuación ha sido implementada en la asignatura Fundamentos Químicos para Ciencia y Tecnología de Alimentos, en el primer curso de dicho grado. Esta asignatura es troncal, de 12 créditos, y está distribuida en tres unidades didácticas: UD1. Introducción a la Química orgánica, UD 2. Química física y UD 3. Cinética y Equilibrios. La DI fue aplicada globalmente a la UD1 y a las prácticas de laboratorio de la UD2 durante el curso 2018-19, en el marco del Proyecto de Docencia Inversa de la UPV. La iniciativa de incorporar la DI a esta asignatura surgió, en cuanto a las PL, por la necesidad de garantizar una adecuada comprensión de sus fundamentos y objetivos, así como de su contextualización en la secuencia de aprendizaje de la asignatura. Por otra parte, en la introducción a la química orgánica, la DI se consideró una alternativa potencialmente útil para conseguir sesiones presenciales más participativas y centradas en los contenidos de mayor exigencia cognitiva, desarrollando los de carácter descriptivo y los relacionados con la formulación y nomenclatura de modo preferentemente no presencial.

En cuanto al tratamiento de las CTs, esta asignatura es punto de control de: "Conocimiento de problemas contemporáneos", donde se pone el acento en el enfoque agroalimentario de la 
UD1 e "Instrumental específica", a la que contribuye el aprendizaje del uso de software químico. Al mismo tiempo, el desarrollo de otras CTs como la organización y gestión del tiempo puede considerarse implícito en la aplicación de la DI.

La aplicación de la DI a las prácticas consistió en una actividad prelaboratorio basada en un vídeo o una actividad con la herramienta Lessons. Esta permite configurar secuencias de aprendizaje cuyos elementos se hallan incorporados o enlazados en un documento digital único, de modo que son fácilmente accesibles al alumnado. En algunos casos se incluyeron también artículos docentes de apoyo para la comprensión de los conceptos o para ampliar algunos de particular interés. En cualquier caso, sus objetivos fueron contextualizar la práctica en cuanto a su fundamentación teórica y en relación al ámbito agroalimentario, así como describir el proceso experimental atendiendo a los fundamentos físico-químicos de las operaciones de laboratorio y los posibles problemas de seguridad. Esta actividad prelaboratorio se complementó con una prueba on-line obligatoria a través de la herramienta "exámenes" de PoliformaT, contribuyendo a la nota final de la práctica. Asimismo, se plantearon actividades post-laboratorio de reflexión y consolidación, susceptibles de ser comentadas en las clases de teoría y preguntadas en el examen. Cabe precisar que las calificaciones de teoría y de las prácticas son independientes, contribuyendo estas últimas (diez en total, para las tres unidades didácticas) en un $25 \%$ a la calificación final.

La UD1, dedicada a la química orgánica, se estructuró en 11 temas elaborados con la herramienta Lessons con la siguiente estructura: (1) Presentación con presentaciones y vídeos de los contenidos descriptivos de menor demanda cognitiva, referidos al contexto agroalimentario, integrando preguntas con feed-back inmediato. Algunos de los vídeos son interactivos, incluyendo ejercicios prácticos. (2) Actividades para la clase presencial, en forma de PDFs o presentaciones, donde se trata la relación estructura-propiedades, isomería, y reactividad, aspectos de mayor demanda cognitiva. En estas sesiones se utilizó ocasionalmente la herramienta Socrative ${ }^{\mathrm{TM}}$ para la evaluación formativa, junto a una autoevaluación on-line semanal. (3) Trabajo en grupos: elaboración de un screencast acerca de un aditivo alimentario. que se evaluó mediante una rúbrica específica. Paralelamente, al comienzo de curso, se propusieron actividades relacionadas con prerrequisitos y sobre el manejo del software ChemSketch $^{\odot}$. También se plantearon durante este periodo inicial actividades no presenciales y presenciales sobre formulación y nomenclatura.

\section{Resultados y discusión}

Para conocer la opinión del alumnado acerca de la metodología implantada (DI), se realizó un sondeo con las siguientes cuestiones en Google forms, al que contestaron 39 estudiantes (44\% del alumnado). Los resultados se muestran en la figura 1. En cuanto a la valoración 
general del modo de abordar los contenidos. (Fig.1a) puede aceptarse que la mayor parte del alumnado (59 \%) valora positivamente la DI. La utilización del material audiovisual (Fig. 1b) registra también un grado de aceptación que avala la calidad del material empleado. Del mismo modo, la valoración de las actividades pre-laboratorio (Fig. 1c) apoya su aplicación generalizada. También es positivamente apreciada la utilidad de la herramienta Lessons (Fig. 1d). En cuanto al modo de introducir la formulación y nomenclatura (Fig. 1e) los resultados también aconsejan no modificar la metodología empleada. También parece apropiado desde la opinión del alumnado mantener la realización de autoevaluaciones (Fig. 1f). La preferencia del alumnado por la explicación presencial del profesorado de los contenidos descriptivos (Fig. 1g) puede explicarse, de acuerdo con Brewer y Movahedazarhouligh (2018) por cierta sensación de desconcierto que diferentes modos de presentar la información pueden producir en el alumnado, sobre todo cuando se enfrenta a la DI al comenzar sus estudios universitarios. Por otra parte, cabría revisar si el diseño y aplicación del material audiovisual es adecuado en cuanto a su duración y densidad semántica. También es necesario garantizar que el alumnado lo utilice correctamente, por lo que puede ser conveniente un seguimiento más riguroso del trabajo no presencial, verificando el uso personal de los vídeos.

Con respecto a la organización general del curso y el seguimiento de los planes de trabajo (Fig. 1h) un $25 \%$ en desacuerdo aconseja la necesidad de revisar la estructura del curso. También cabe reflexionar acerca de la autopercepción que el alumnado tiene de su organización personal del tiempo (Fig. 1i). Aproximadamente el 54\% está de acuerdo en que no se organiza bien, aunque un $25 \%$ piensa que ese no es el problema. Este resultado puede explicarse en parte por tratarse de alumnado de primer curso. La organización y gestión del tiempo (una de las competencias transversales en el proyecto de la UPV) es especialmente sensible a la madurez del alumnado y consecuentemente, a su capacidad de reflexión y autocrítica. Sin embargo, hay otro factor que puede considerarse decisivo: el hecho de que la DI se aplique solo puntualmente en ciertas materias. Es evidente el impacto que los actos de evaluación de otras asignaturas producen en la DI, principalmente en el trabajo no presencial. 

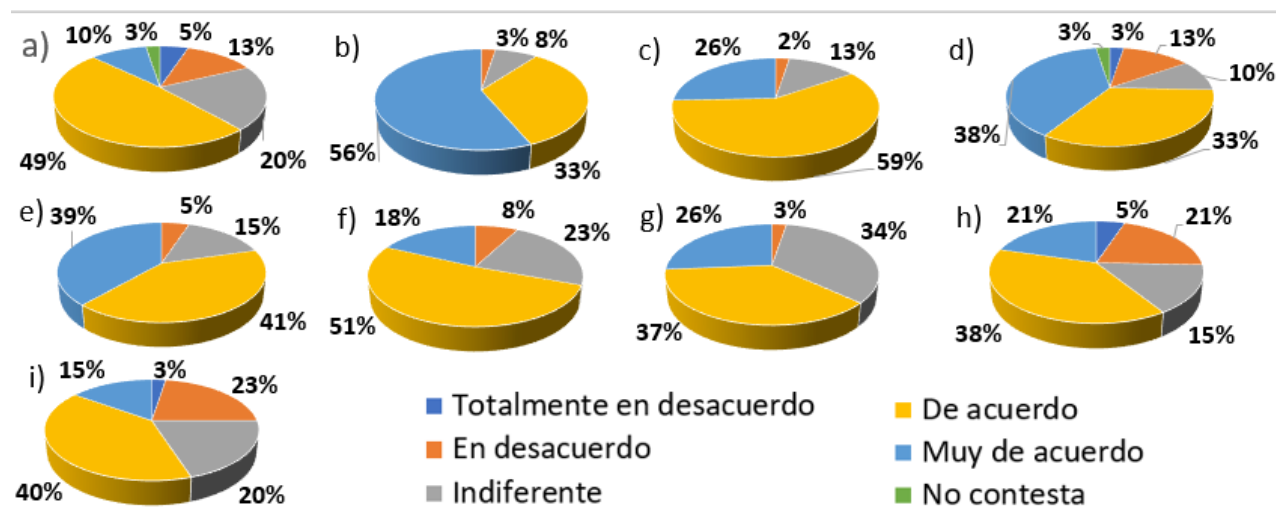

Fig.1. Opiniones de los estudiantes sobre la implantación de la DI en la asignatura. Se muestra en diagrama de sectores, representados por colores ( $n^{o}$; \%), las opiniones sobre las cuestiones: a) En general, me parece apropiado el modo de abordar los contenidos. b) La utilización del material audiovisual (polimedias, principalmente) me ha resultado útil en el desarrollo de la asignatura. c) Las actividades pre-laboratorio contribuyen a mejorar las sesiones de prácticas y a comprender mejor su fundamento y objetivos. d) La herramienta de poliformaT "Lessons" la considero un importante avance a la hora de organizar los contenidos de la asignatura. e) El modo de introducir la formulación y nomenclatura (sesiones iniciales de introducción y ejercicios resueltos a lo largo de los temas) lo considerado adecuado. f) La realización de autoevaluaciones me ha resultado útil para comprender mejor los conceptos introducidos en la asignatura. g) En los contenidos descriptivos, como las aplicaciones agroalimentarias, he echado de menos la explicación en clase. h) La organización general del curso: exámenes, trabajos, etc., me impide ir al día, no realizando las tareas indicadas en los planes de trabajo. i) Una mejor organización personal del tiempo me ayudaría a llevar la asignatura al día, realizando en su momento las tareas indicadas en los planes de trabajo.

En cuanto a los resultados académicos, la Fig. 2 muestra de modo comparativo los resultados de cada uno de los bloques del examen y la calificación global de la UD1, (que integra el trabajo complementario y los resultados de la evaluación formativa), para el curso 2018-19 y 2017-18, en el que no se aplicó la DI. Excepto en reactividad, se observan pequeños incrementos que son significativos ( $\mathrm{P} \leq 0.05$, prueba " $\mathrm{t}$ " de Student), en los bloques de isomería (típicamente presencial) y formulación (desarrollado de modo no presencial). Sin embargo, donde el cambio de enfoque docente fue más drástico (aspectos descriptivos), totalmente no presencial, el rendimiento fue prácticamente idéntico. Una mejoría relativamente importante, aunque no llega a ser estadísticamente significativa, se ha observado en el apartado de propiedades físicas y estructura molecular. En cuanto a la calificación global, la DI muestra una media significativamente mayor $(\mathrm{P}=0.05)$. Comparando la calificación media de la UD1 del curso 2018-19 con las obtenidas en la UD2 (5.29) y UD3 (5.37) en las que no se aplicó la DI (en cuanto a contenidos teóricos), se observó una cierta mejoría que no llega a ser significativa para $\mathrm{P} \leq 0.05$ ( $\mathrm{P}=0.08$ en ambos casos $)$. 


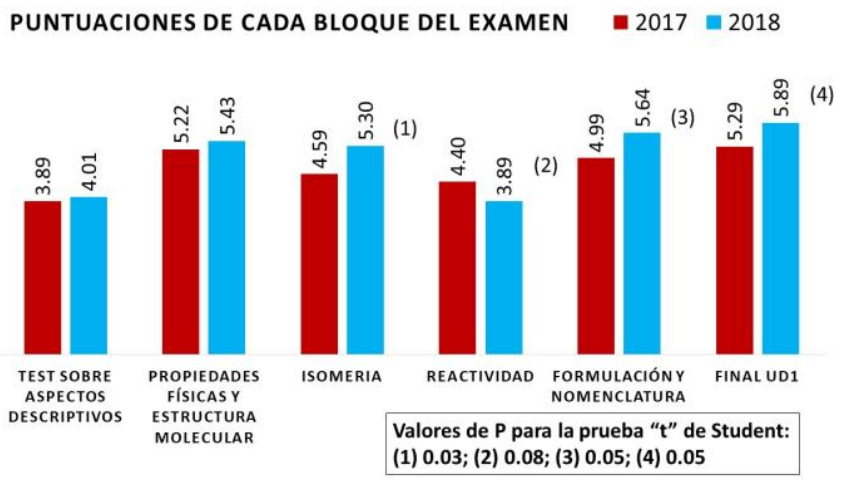

Fig.2. Calificaciones de los diferentes bloques del examen parcial de la UD1 y nota global de los cursos 2017 y 2018. Se muestran los valores de P para la prueba " $t$ " de Student cuando pueden considerarse relevantes.

\section{Conclusiones}

En general, la DI es positivamente valorada por el alumnado encuestado en cuanto a los recursos utilizados. Aspectos tales como el material audiovisual, empleo de la herramienta Lessons o la realización de autoevaluaciones han sido muy bien valorados, del mismo modo que la aplicación de la DI a las actividades pre-laboratorio. Estas han sido un recurso muy útil para la mejora de las prácticas teniendo en cuenta su contenido, número de estudiantes y duración. Todos estos resultados pueden considerarse satisfactorios tratándose del primer año de experimentación con alumnado de primer curso. Es la percepción global de la DI lo que parece más susceptible de mejora, siendo evidente la necesidad de explicar más detenidamente al alumnado esta metodología al principio del curso, así como de proporcionarle pautas y orientaciones para mejorar la organización y gestión del tiempo, competencia transversal que juzgamos clave en la implementación de la DI. Por último, debe constatarse que, pese a haber constituido de una experiencia claramente positiva tanto para el profesorado y el alumnado, un impacto claramente positivo de la DI en el aprendizaje exige, si no su aplicación en todas las asignaturas de un curso, al menos una coordinación entre ellas que evite interferencias en el trabajo no presencial, aspecto clave de la DI.

\section{Referencias}

Abeysekera, L., \& Dawson, P. (2015). Motivation and cognitive load in the flipped classroom: definition, rationale and a call for research. Higher Education Research \& Development, 34(1), 114. 
Akçayır, G., \& Akçayır, M. (2018). The flipped classroom: A review of its advantages and challenges. Computers \& Education, 126, 334-345.

Brewer, R., \& Movahedazarhouligh, S. (2018). Successful stories and conflicts: A literature review on the effectiveness of flipped learning in higher education. Journal of Computer Assisted Learning, 34(4), 409-416.

Clark, J. M., \& Paivio, A. (1991). Dual coding theory and education. Educational Psychology Review, 3(3), 149-210.

Cormier, C., \& Voisard, B. (2018). Flipped classroom in Organic chemistry has significant effect on students' grades. Frontiers in ICT, 4, 30.

Galicia-Alarcón, L. A., \& Edel-Navarro, J. A. B. T. (2014). Revisión del modelo atención, relevancia, confianza y satisfacción (ARCS). Los Modelos Tecno-Educativos, 47.

Jensen, J. L., Holt, E. A., Sowards, J. B., Ogden, T. H., \& West, R. E. (2018). Investigating strategies for pre-class content learning in a flipped classroom. Journal of Science Education and Technology, 27(6), 523-535.

O'Flaherty, J., \& Phillips, C. (2015). The use of flipped classrooms in higher education: A scoping review. The internet and higher education, 25, 85-95.

Proyecto Docencia Inversa UPV. https://docenciainversa.blogs.upv.es/el-proyecto/proyecto-claseinversa-upv/ (Consulta: 18-5-2019).

Ryan, M. D., \& Reid, S. A. (2015). Impact of the flipped classroom on student performance and retention: A parallel controlled study in general chemistry. Journal of Chemical Education, 93(1), $13-23$.

¿Qué es Polimedia? https://www.upv.es/entidades/ASIC/catalogo/522359normalc.html (Consulta: 2105-2019)

Teo, T. W., Tan, K. C. D., Yan, Y. K., Teo, Y. C., \& Yeo, L. W. (2014). How flip teaching supports undergraduate chemistry laboratory learning. Chemistry Education Research and Practice, 15(4), 550-567.

Tucker, B. (2012). The flipped classroom. Education next, 12(1), 82-83. 